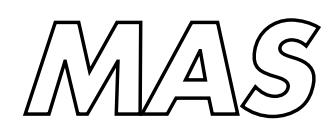

Modelling, Analysis and Simulation

\footnotetext{
Modelling, Analysis and Simulation

MAS Efficient Computation of Steady, 3D Water-Wave Patterns
}

\author{
M. Lewis, B. Koren
}

Report MAS-e0322 December 16, 2003 
CWI is the National Research Institute for Mathematics and Computer Science. It is sponsored by the Netherlands Organization for Scientific Research (NWO).

CWI is a founding member of ERCIM, the European Research Consortium for Informatics and Mathematics.

CWI's research has a theme-oriented structure and is grouped into four clusters. Listed below are the names of the clusters and in parentheses their acronyms.

Probability, Networks and Algorithms (PNA)

Software Engineering (SEN)

Modelling, Analysis and Simulation (MAS)

Information Systems (INS)

Copyright (C) 2003, Stichting Centrum voor Wiskunde en Informatica

P.O. Box 94079, 1090 GB Amsterdam (NL)

Kruislaan 413, 1098 SJ Amsterdam (NL)

Telephone +31205929333

Telefax +31205924199

ISSN 1386-3703 


\title{
Efficient Computation of Steady, 3D Water-Wave Patterns
}

\begin{abstract}
Numerical methods for the computation of stationary free surfaces is the subject of much current research in computational engineering. The present report is directed towards free surfaces in maritime engineering. Of interest here are the long steady waves generated by ships, the gravity waves. In the present report an existing 2D iterative method for the computation of stationary gravity-wave solutions is extended to 3D, numerically investigated, and improved. The method employs the so-called quasi free-surface boudary condition. As test cases we cosider gravity-wave patterns due to pressure perturbations imposed at the free surface of a steady, uniform horizontal flow. The effects are studied of the distance of the imposed pressure distribution to the far-field boundary, the magnitude of the imposed pressure perturbation, and the mesh widths. In all experiments, our focus is on the convergence behavior of the free-surface iteration process.
\end{abstract}

2000 Mathematics Subject Classification: 76-xx

Keywords and Phrases: incompressible Navier-Stokes equations; free-surface flows 


\title{
EFFICIENT COMPUTATION OF STEADY, 3D WATER-WAVE PATTERNS
}

\author{
Mervyn Lewis \\ CWI, P.O. Box 94079, 1090 GB Amsterdam, The Netherlands \\ Barry Koren \\ CWI and Delft University of Technology, Faculty of Aerospace Engineering, \\ P.O. Box 5058, 2600 GB Delft, The Netherlands, \\ Senior member AIAA.
}

\begin{abstract}
$\underline{\text { ABSTRACT }}$
Numerical methods for the computation of stationary free surfaces is the subject of much current research in computational engineering. The present report is directed towards free surfaces in maritime engineering. Of interest here are the long steady waves generated by ships, the gravity waves. In the present report an existing 2D iterative method for the computation of stationary gravity-wave solutions is extended to $3 \mathrm{D}$, numerically investigated, and improved. The method employs the so-called quasi freesurface boundary condition. As test cases we consider gravity-wave patterns due to pressure perturbations imposed at the free surface of a steady, uniform horizontal flow. The effects are studied of the distance of the imposed pressure distribution to the far-field boundary, the magnitude of the imposed pressure perturbation, and the mesh widths. In all experiments, our focus is on the convergence behavior of the free-surface iteration process.
\end{abstract}

\section{INTRODUCTION}

Examples of free-surface problems in science and engineering are vast. The application we are aiming at are the water-wave patterns generated by a ship hull moving with a steady, rectilinear velocity.

The inherent difficulty of computing free-surface flows is the interdependence of the free-surface location and the unknowns of the flow problem. Numerical techniques available for the computation of free-surface flows can be divided into two categories: the fixed-grid (Eulerian) and the moving-grid (Lagrangian) methods. See the introduction of ${ }^{7}$ for a general overview of these methods. In the case of a smooth free surface without overturning waves, i.e., when the free surface can be represented by a height function, surface-fitting methods are unsurpassed in accuracy. Because our primary focus is on the computation of non-overturning gravity waves, this method is adopted here. Furthermore, a decision has to be made on the formulation of the problem, i.e., time-dependent versus stationary. If the goal is to solve for the steady-state solution, the usual time-integration methods are computation- ally inefficient, see e.g. ${ }^{1,3}$. This is due to the fact that the convergence to steady state is retarded by slowly attenuating transient surface gravity waves. It can be shown that the attenuation behaves as $\mathcal{O}\left(t^{(1-d) / 2}\right)$ in $\mathbb{R}^{d}$, see e.g. ${ }^{2}$. This specific transient behaviour in combination with the time-step restriction, in case of explicit methods, renders this method prohibitively expensive in actual computations. Several improvements have been suggested, such as pseudo-time integration and quasi-steady methods, e.g. ${ }^{4,10}$.

The main topic of this work is the investigation of the convergence behavior for 3D problems, of a new nonmonolithic (i.e., an alternating or partitioned) free-surface iteration method, proposed and worked out in ${ }^{2}$ for 2D flows. In ${ }^{2}$, the so-called quasi free-surface condition (QFSC) is derived. This nonlinear free-surface boundary condition plays an essential role in the new free-surface iteration method. In the present paper, this boundary condition is extended to $3 \mathrm{D}$ and the free-surface iteration method is applied to a test case involving stationary gravity waves induced by a pressure perturbation imposed at the free surface of a 3D water flow. Varying the amplitude of the perturbation alters the nonlinearity of the resulting wave system. Each step of the free-surface iteration method involves the solution of a stationary Navier-Stokes boundary-value problem. The numerical results are compared with a solution of the potential-flow method from ${ }^{9}$.

The contents of this paper is the following. In Section 2 , the governing equations are introduced. Section 3 describes the computational method, in particular the new stationary free-surface iteration method. This method involves approximating the solution of a (sequence) of steady Navier-Stokes boundary-value problems. In Section 4, various numerical results are presented for the freesurface algorithm. Section 5 concludes this paper.

\section{GOVERNING EQUATIONS}

In this section an outline is given of the mathematical model which describes viscous free-surface flows. The first subsection lists the equations which describe the fluid flow, the next subsection treats the free-surface boundary conditions and the last the quasi free-surface condition. 


\section{FLOW EQUATIONS}

Let $\Omega(\mathbf{x}) \subset \mathbb{R}^{3}$ be the physical domain which is occupied by the fluid and let us split the domain boundary as $\partial \Omega=\Gamma_{\mathrm{FS}} \cup \Gamma_{0}$, where $\Gamma_{\mathrm{FS}}$ denotes the free surface and $\Gamma_{0}$ the remaining (fixed) part of $\partial \Omega$. Positions in $\mathbb{R}^{3}$ are identified with respect to a Cartesian coordinate system $\mathbf{e}_{\alpha}, \alpha=\{x, y, z\}$ with $\mathbf{g}=-g \mathbf{e}_{z}$ denoting the gravity force. The state of the flow is characterized by the velocity field $\mathbf{u}(\mathbf{x}): \Omega \rightarrow \mathbb{R}^{3}$ and the pressure $p(\mathbf{x}): \Omega \rightarrow \mathbb{R}$ and is governed by

$$
\left\{\begin{array}{l}
\nabla \cdot\left(\mathbf{u u}^{T}\right)+\nabla \varphi-\operatorname{Re}^{-1} \tilde{\Delta} \mathbf{u}=\mathbf{0}, \quad \forall \mathbf{x} \in \Omega, \\
\nabla \cdot \mathbf{u}=0, \quad \forall \mathbf{x} \in \Omega,
\end{array}\right.
$$

where $\operatorname{Re}=U \ell / \nu$ is the Reynolds number, with $U$ and $\ell$ a reference speed and length and with $\nu$ the kinematic viscosity. The sole external force, gravity, can be incorporated into the pressure by redefining it as

$$
\varphi(\mathbf{x}):=p(\mathbf{x})+\operatorname{Fr}^{-2} z,
$$

with $\mathrm{Fr} \equiv U / \sqrt{g \ell}$ being the Froude number. It is furthermore assumed that diffusion in main flow direction, say the $x$-direction, can be safely neglected. As a result the viscous term reduces to $\tilde{\Delta}=\partial_{y}^{2}+\partial_{z}^{2}$, which in turn reduces the number of boundary conditions to be imposed at the $x$-outlet boundary.

\section{FREE-SURFACE CONDITIONS}

The free-surface boundary conditions follow from the general interface conditions and the assumptions that both density and viscosity of one of the adjacent fluids vanish at the interface and that the interface is impermeable. In many applications of interest, especially in those which admit steady solutions, the free surface can be expressed as a single-valued height function $\eta: \Gamma_{\mathrm{FS}}=\{(\mathbf{x}): z=$ $\eta(x, y)\}$. Impermeability leads to the steady form of the kinematic condition

$$
\mathbf{u} \cdot \nabla \eta(x, y)=\mathbf{u} \cdot \mathbf{e}_{z} .
$$

This formulation imposes smoothness restrictions on the shape of the free surface. Vanishing interfacial stresses result in three dynamic conditions, namely

$$
p(\mathbf{x})-2 \operatorname{Re}^{-1} \frac{\partial \mathbf{u}_{n}}{\partial \mathbf{n}}=p_{\mathrm{FS}}(\mathbf{x}),
$$

in the direction normal to the free surface, with $p_{\mathrm{FS}}(\mathbf{x})$ the imposed pressure distribution along the free surface, and

$$
\mathbf{t}^{(\alpha)} \cdot \tau(\mathbf{u}) \cdot \mathbf{n}=0, \quad \alpha=1,2
$$

tangential to the free surface, where $\tau(\mathbf{u})$ is the viscous stress tensor for an incompressible fluid. Here $\left(\mathbf{n}, \mathbf{t}^{(\alpha)}\right), \alpha=1,2$ are the unit normal vector and the orthonormal tangential vectors, respectively. For the practical application envisaged here, the viscous contribution to the normal dynamic condition may be neglected, resulting in an inhomogeneous Dirichlet condition for the pressure $p(\mathbf{x})$. It has been assumed that surface tension effects can be safely ignored.

\section{THE QUASI FREE-SURFACE CONDITION}

In free-surface flows, an interdependence exists of the state variables $(\mathbf{u}(\mathbf{x}), p(\mathbf{x}))$ and their spatial domain, through both the kinematic and dynamic conditions. In general, the free-surface flow problem is stated by equations (1) subject to (3)-(5) on $\Gamma_{F S}$, together with additional boundary conditions on $\Gamma_{0}$. Note that the number of free-surface conditions is one more than the number of boundary conditions allowed to be imposed on a boundary in a Navier-Stokes boundary-value problem. Many concurrent free-surface iteration methods employ a kinematic free-surface iteration process, i.e., a method in which they first solve (1) subject to the dynamic conditions at an approximate location of the free surface. In the following step, they adjust the free surface using the kinematic condition. Results obtained with these methods can be found in, e.g., 1,3 for the fully time-dependent approach and in, e.g., ${ }^{4,10}$ for pseudo-time integration and quasi-steady methods.

However, it is important to note that any combination of three conditions from (3) - (5) is also allowed as boundary condition for the Navier-Stokes boundary-value problem, leaving the fourth condition to be used to locate the freesurface. Here we apply an iteration method based on the use of the quasi free-surface boundary condition (QFSC), which, in unsteady form reads

$$
\frac{\partial \varphi}{\partial t}+\mathbf{u} \cdot \nabla \varphi-\operatorname{Fr}^{-2} \mathbf{u} \cdot \mathbf{e}_{z}=\mathbf{u} \cdot \nabla p_{\mathrm{FS}}
$$

Here we will only apply the steady formulation of the QFSC. The QFSC is a result of the combination of the kinematic- and the (normal) dynamic free-surface condition. Use of this special free-surface condition has the advantage that it does not decouple the kinematic and dynamic free-surface conditions. In fact, it is the combination of the kinematic and dynamic condition which yields the wave-like solutions. A more formal derivation of this boundary condition, in $\mathbb{R}^{2}$, can be found in ${ }^{2}$.

\section{COMPUTATIONAL METHOD}

It has been argued in the introduction that time-dependent formulations are computationally inefficient for obtaining a steady-state solution. Therefore, we directly address the steady form of the governing equations. We will first state our new free-surface iteration method, which requires the solution of a (sequence) of steady Navier-Stokes boundary-value problems. This will be briefly described in the last part of this section.

Denoting (1) as $\mathcal{N S}(\mathbf{u}, \varphi)=0$, and the boundary conditions to be imposed at $\Gamma_{0}$ as $\mathcal{B}(\mathbf{u}, \varphi)=0$, the solution of the free-surface flow problem can be found by iterating the following two steps:

I. For a given boundary $\Gamma_{\mathrm{FS}}$, solve $(\mathbf{u}, \varphi)^{T}$ from

$$
\begin{aligned}
& \mathcal{N} \mathcal{S}(\mathbf{u}, \varphi)=0, \quad \forall \mathbf{x} \in \Omega, \\
& \mathcal{B}(\mathbf{u}, \varphi)=0, \quad \forall \mathbf{x} \in \Gamma_{0},
\end{aligned}
$$




$$
\begin{aligned}
& \left.\begin{array}{c}
\mathbf{t}^{(\alpha)} \cdot \tau(\mathbf{u}) \cdot \mathbf{n}=0, \quad \alpha=1,2 \\
\mathbf{u} \cdot \nabla \varphi-\mathrm{Fr}^{-2} \mathbf{u} \cdot \mathbf{e}_{z}=\mathbf{u} \cdot \nabla p_{\mathrm{FS}}
\end{array}\right\}, \quad \forall \mathbf{x} \in \Gamma_{\mathrm{FS}} . \\
& \text { If }\left\|p-p_{\mathrm{FS}}\right\|>\epsilon_{\mathrm{FS}}, \text { then do step II, else stop. }
\end{aligned}
$$

II. Use the solution $(\mathbf{u}, \varphi)^{T}$ of I to obtain a new approximation of $\Gamma_{\mathrm{FS}}$ according to

$$
\left\{\left(x, y, z:=\operatorname{Fr}^{2}\left(\varphi(\mathbf{x})-p_{\mathrm{FS}}(\mathbf{x})\right): \forall \mathbf{x} \in \Gamma_{\mathrm{FS}}\right\},\right.
$$

next return to step I.

where $\epsilon_{\mathrm{FS}}$ is some specified tolerance.

Note that if $\Gamma_{\mathrm{FS}}$ is the true free surface then the normal dynamic condition (i.e. $p=p_{\mathrm{FS}}$ ) is satisfied. In that case $\mathbf{n} \| \nabla p$, and (6) implies that the solution of step I satisfies the kinematic and tangential dynamic conditions. Hence step I yields the free-surface flow then. It is important to note that steps I and II do not contain any time derivative and as a result do not suffer from the slow decay rate of the transient waves normally encountered in time-dependent methods.

The reduced Navier-Stokes equations and boundary conditions are discretized using a collocated, second- order accurate finite-difference method. The resulting system of nonlinear algebraic equations is solved by Newton's method. The linear system is solved by adopting a space-marching Gauss-Seidel algorithm with the marching performed in the main flow direction. This approach is natural in view of the strong parabolic nature of the flow equations. The space-marching procedure yields a size reduction of the linear-algebra problem. The smaller linear systems are solved using a CILU(0) preconditioned Krylov-subspace method (GMRES). The convergence criterion for the solution of the Newton iteration process is that the change in the pressure, measured in the infinitynorm, is smaller than a specified tolerance. More details can be found in ${ }^{5}$.

\section{NUMERICAL RESULTS}

Here we present some numerical results obtained with the above described method. At the free surface we impose a Gaussian pressure distribution

$$
p_{\mathrm{FS}}:=P e^{-\alpha\left(\left(x-x_{c}\right)^{2}+\left(y-y_{c}\right)^{2}\right)}, \quad P, \alpha \in \mathbb{R}^{+},
$$

where $P$ and $\alpha$ determine the strength of the perturbation and hence the nonlinearity of the resulting wave system.

As a first test case we consider the following parameter values for the Gaussian pressure perturbation (10): $P=0.05, \alpha=4, \mathrm{Fr}=0.6$ and $\left(x_{c}, y_{c}\right)=(0,0)$. This is conform to computations done with the potentialflow method described in ${ }^{11}$. The Reynolds number is set equal to $\operatorname{Re}=10^{6}$. The current computation is performed on the basis mesh, $\Omega_{h}$, which has 81,31 and 31 nodes in the $x$-, $y$ - and $z$-direction, respectively. $\Omega_{h}$ is constructed such that the Kelvin wedge, which bounds the spatial distribution of the wave energy, does not intersect the external boundary by taking $x_{\max }=6$ and $y_{\max }=3$. The wedge makes a semi-angle of $19.5^{\circ}$ with the main flow direction. (See Section 3.10 in ${ }^{8}$ for a derivation of this result.) Other boundary coordinates chosen are $x_{\min }=-2$ and $z_{\min }=-3$. For all computations, the initial estimate of the free surface is the plane $z=0$.

As mentioned before, our focus is on the convergence behavior of the new free surface iteration method. We distinguish two iteration processes: an outer and an inner iteration process, the iteration processes II and I, respectively, as described in the previous section. The convergence of the outer iteration, the free-surface method, is measured through the pressure defect at the free surface. The convergence of the inner iteration, the Navier-Stokes method, is measured by computing $\|\mathbf{R}\|_{\infty}$, where $\mathbf{R}$ is the residual of the flow equations (1). For the present test case, the convergence behavior of the inner iteration is shown in the left graph of Figure 1.

The two large jumps in the residual (at about $n=40$ and $n=80$ ) are due to free-surface updates. After each free-surface update, the residuals are scaled, which explains the identical residual values after these updates. Figure 1 reveals that the inner-iteration process on the mesh obtained after the third free-surface update starts to oscillate with an increasing amplitude, preventing further decrease of the residuals. A closer inspection has shown that these residuals occur at a location near the outflow boundary, in the first grid plane underneath the free surface. This indicates a local incompatibility between the free-surface flow and the underlying bulk-flow solution. The proposed remedy will be addressed in the next section. The convergence of the free-surface iteration process is monitored through the pressure defect $\left\|p^{n}-p_{\mathrm{FS}}\right\|$, measured in some usual norms. Here $p^{n}=\varphi^{n}-\mathrm{Fr}^{-2} z^{n}$ is the hydrodynamic pressure minus the hydrostatic part. The decrease in the pressure defect is shown in the right graph of Figure 1. The free-surface iteration appears to converge very fast; the second and third free-surface updates are already negligible as compared to the first, as can be seen in the left graph of Figure 2.

In Figure $2, \zeta=\eta / \eta_{\max }$ is the wave elevation divided by the maximum obtainable elevation $\eta_{\max }=\frac{\mathrm{Fr}^{2}}{2}$. The last iterate in the left graph of Figure 2 shows a wave length of $\lambda=2.3$ and a maximum scaled amplitude of about $15 \%$. These results correspond fairly well with the results obtained through the potential-flow method described in ${ }^{11}$. For further comparison purposes a solution for this test case has also been computed through the potential-flow method described in ${ }^{9}$. The corresponding wave pattern is shown in the right graph of Figure 2, together with the present Navier-Stokes solution (the dotted line). Differences between both wave patterns are to be attributed to differences in the two continuous models as well as their numerical discretizations. In Figure 3 we still show the entire Navier-Stokes wave pattern as obtained after the third free-surface update.

\section{EFFECT OF THE OUTFLOW BOUNDARY CONDITION}

The incipient divergence of the inner iteration on the third mesh, as shown in Figure 1, is due to an incompatibil- 
ity between the free-surface flow solution and the underlying bulk-flow boundary condition at the outflow boundary. This discrepancy can be overcome by extending $\Omega_{h}$ with a wave-dissipation zone, see, e.g., ${ }^{6}$. Such a zone is added to rapidly dissipate all the wave energy from the numerical solution, hereby reducing the solution to uniform flow conditions.

The wave energy is better dissipated by increasing the numerical viscosity. This is achieved by $(i)$ reducing the accuracy of the discretization of (6) in the wavedissipation zone to first order, and $(i i)$ by applying grid stretching in the wave-dissipation zone. The grid-point distribution in the wave-dissipation zone is controlled by

$$
x_{i}=x_{\max } e^{\beta \frac{\Delta x}{x_{\max }} i}, \quad y_{j}=y_{\max } e^{\beta \frac{\Delta y}{y_{\max }} j},
$$

with

$$
i=1, \ldots,\left(N_{x}\right)_{\mathrm{add}}, \quad j=1, \ldots,\left(N_{y}\right)_{\mathrm{add}},
$$

where $\beta$ is the stretching factor in $x$ - and $y$-direction, and where $\left(N_{x}\right)_{\text {add }}$ and $\left(N_{y}\right)_{\text {add }}$ are the numbers of additional points in both directions. Both numbers are fixed by specifying the maximally allowable mesh width, e.g., $\left|x_{\left(N_{x}\right)_{\text {add }}}-x_{\left(N_{x}\right)_{\text {add }}-1}\right|$ is set at 0.5 . The same is done for the $y$-direction. The extended mesh has $149 \times 53 \times 31$ points. In absence of a wave solution near the outflow plane the incompatibility between the free-surface flow and the bulk flow disappears and a homogeneous Neumann condition for the pressure can be specified as outflow boundary condition.

On this extended domain we computed five free-surface updates. The positive influence of the wave-dissipation zone on the convergence behavior of both the inner and outer iteration can be clearly seen when comparing Figure 4 with Figure 1.

In Figure 5, we show the entire wave pattern as obtained after the fifth free-surface update. The wave damping in the dissipation zone, which starts at $x=10$, is clearly visible.

In Figure 6, we still depict the elevations after the first and fifth free-surface update, in the planes $y=0$ and $y=2$. Particularly from the left graph in Figure 6 , it appears that the free-surface iteration converges very fast. (The initial estimate for the free surface is the line $\zeta=0$.) Note that, as opposed to the first iterate in the left graph of Figure 6 and as opposed to the three iterates in the left graph of Figure 2, the free surface in the symmetry plane, as obtained after the fifth update, shows a small trough at about $x=-1$. The trough can still be observed at $y=2$ (the right graph of Figure 6).

In Figure 7 we plotted our Navier-Stokes wave patterns in the symmetry plane, as obtained on the domains with and without wave-dissipation zone. For comparison purposes, in Figure 7 we also give the wave pattern obtained with the potential-flow method described in ${ }^{9}$. Differences between the two Navier-Stokes wave patterns in Figure 7 may be attributed mainly to the fact that the solution on the domain without wave-dissipation zone is less far converged than that on the domain with wave-dissipation zone. Still concerning Figure 7, note the strong wave damping starting from $x=10$, the $x$-coordinate of the upstream boundary of the wave-dissipation zone.

\section{MESH-WIDTH STUDY}

In this section, the effects of the mesh width on the convergence and accuracy of the solution are investigated. This is done by comparing the numerical results obtained for $\Omega_{h}$ with results obtained for $\Omega_{2 h}$ and $\Omega_{\frac{h}{2}}$. To reduce the influence of the outflow boundary condition a wave-dissipation zone is added to each of the three grids.

The convergence of the inner iteration on $\Omega_{2 h}$ and $\Omega_{\frac{h}{2}}$ is shown in Figure 8. It appears that the strategy for the solution of the Navier-Stokes subproblems is not (yet) optimally efficient. To further illustrate this, in Figure 9, we have plotted $n_{\text {inner }}$, the number of inner iterations needed for the first Navier-Stokes subproblem, versus $N_{x}$, the number of grid points in $x$-direction. A least-squares fit reveals a linear dependence of $n_{\text {inner }}$ on $N_{x}$. (Ideally, $n_{\text {inner }}$ is independent of $N_{x}$; this may be realized with a proper multigrid method.)

For the three grids, the wave elevations in the plane $y=0$ are shown in Figure 11. They show a clear dependence of the wave length and wave amplitude on the mesh width. These effects can be attributed to the discretization of the quasi free-surface boundary condition. In (6), $\mathbf{u} \cdot \nabla p$ is discretized using the $\mathcal{O}\left(h^{2}\right)$ upwind scheme. The meshwidth dependence can be understood through a spectral analysis of (6). The dominant term in (6) is $u p_{x}$; for first analysis purposes, (6) is reduced to $u p_{x}=0$. For $u$ positive, the corresponding modified equation reads

$$
u \frac{\partial p}{\partial x}=u \frac{h^{2}}{3} \frac{\partial^{3} p}{\partial x^{3}}-u \frac{h^{3}}{4} \frac{\partial^{4} p}{\partial x^{4}}+\mathcal{O}\left(h^{4}\right) .
$$

Inserting a single wave solution of the form $p=P e^{\mathrm{i} k x}$, the spectral representation of the leading term results in

$$
u \mathrm{i} k\left(1+\frac{1}{3} h^{2} k^{2}\right) P e^{\mathrm{i} k x}=\mathcal{O}\left(h^{3}\right) .
$$

From this relation it can be concluded that the numerical advection velocity of $p$ increases with increasing mesh width, thus increasing the length of the gravity wave. The latter increase can be explained from the dispersion relation for waves on deep water, see ${ }^{8}$. Particularly when applying a multigrid solution strategy, one should be aware of the mesh dependency of the wave lengths.

\section{Higher-order Discretization OF THE QFSC}

As a next step, we replace the $\mathcal{O}\left(h^{2}\right)$ upwind discretization of $\nabla \varphi$ in (6) by an $\mathcal{O}\left(h^{3}\right)$ upwind scheme. Then, the modified equation reads

$$
u \frac{\partial p}{\partial x}=-u \frac{h^{3}}{12} \frac{\partial^{4} p}{\partial x^{4}}+\mathcal{O}\left(h^{4}\right),
$$

showing no dispersion error, but a fourth-order dissipation error, which is responsible for a decrease of the wave 
elevation. Note that the fourth-order dissipation error is smaller than that of (13). A comparison of the solution obtained with the two schemes, after a single freesurface update, is shown in Figure 12, together with the results obtained through the potential-flow method described in ${ }^{9}$. As expected, the $\mathcal{O}\left(h^{3}\right)$ scheme gives slightly higher waves. But it also yields a slightly less fast convergence of both the inner and outer iteration than the $\mathcal{O}\left(h^{2}\right)$ scheme (compare Figure 13 and Figure 4). In the remainder of this paper we do not use the $\mathcal{O}\left(h^{3}\right)$ discretization of the quasi free-surface boundary condition.

\section{CASE WITH INCREASED AMPLITUDE}

The present numerical study concerns the computation of the wave pattern on $\Omega_{h}$, for a stronger imposed pressure perturbation. The amplitude of the perturbation is increased from $P=0.05$ to $P=0.2$, leaving the other parameters unaltered. The convergence history of the inner iteration is shown in the left graph of Figure 14. The computed wave elevation, in the plane of symmetry, is shown in the right graph of Figure 14. This figure shows that the deepest trough has fallen off to approximately $\zeta=-0.88$, instead of $\zeta=-0.18$ for the $P=0.05$ case, which indicates that the wave system behaves nonlinearly.

\section{CONCLUSIONS}

Solution of the steady, free-surface Navier-Stokes equations through a time-stepping approach is known to be inefficient, particularly in 3D. Recently, for the 2D, freesurface Navier-Stokes equations, Van Brummelen et al. have proposed a non-monolithic free-surface algorithm that does not follow a time-stepping approach. In the present paper we have extended this algorithm to 3D and have applied it to free-surface flow problems with a varying degree of nonlinearity.

Our results show that, for convergence purposes, it makes sense (i) to take the computational domain sufficiently large and (ii) to discretize the quasi free-surface boundary condition only first-order accurate in the far field. (In this way, unperturbed far-field boundary conditions can be imposed.)

The considered 3D free-surface algorithm appears to quickly yield the proper 3D wave physics. The freesurface pressure defect appears to converge almost gridindependently. For linear and mildly nonlinear wave systems, free-surface iteration may not even be necessary though; only a single free-surface update may be sufficient for finding the wave pattern to within engineering accuracy.

\section{ACKNOWLEDGMENT}

The authors would like to acknowledge dr. Hoyte Raven of MARIN for his useful comments.

This work was supported by the Dutch Technology Foundation STW.

\section{REFERENCES}

1 Alessandrini B, Delhommeau G. Simulation of threedimensional unsteady viscous free surface flow around a ship model. International Journal for Numerical Methods in Fluids 1994; 19: 321-342.

2 van Brummelen EH, Raven HC, Koren B. Efficient numerical solution of steady free-surface Navier-Stokes flow. Journal of Computational Physics 2001; 174: 120-137.

3 Campana E, Di Mascio A, Esposito PG, Lalli F. Viscous-inviscid coupling in free surface ship flows. International Journal for Numerical Methods in Fluids 1995; 21: 699-722.

4 Farmer J, Martinelli L, Jameson A. A fast multigrid method for the nonlinear ship wave problem with a free surface. In Proceedings of the Sixth International Conference on Numerical Ship Hydrodynamics, Patel W, Stern F (eds); National Academy Press: Washington D.C., 1994; pp. 155-172.

${ }^{5}$ Hoekstra M. Numerical simulation of ship stern flows with a space-marching Navier-Stokes method. PhD thesis, Delft University of Technology: Delft, 1999.

6 Israeli M, Orszag S. Approximation of radiation boundary conditions. Journal of Computational Physics 1981; 41: 115-135.

7 Koren B, Lewis MR, van Brummelen EH, van Leer B. Riemann-problem and level-set approaches for two-fluid flow computations, I. Linearized Godunov scheme. Report MAS-R0112, CWI: Amsterdam, 2001.

8 Lighthill MJ. Waves in Fluids. Cambridge University Press: Cambridge, 1958.

9 Raven HC. A solution method for the nonlinear ship wave resistance problem. PhD thesis, Delft University of Technology: Delft, 1996.

10 Tzabiras GD. A numerical investigation of 2D, steady free surface flows. International Journal for Numerical Methods in Fluids 1997; 25: 567-598.

11 Wyatt DC. Development and assessment of a nonlinear wave prediction methodology for surface vessels. Journal of Ship Research 2000; 44: 96-107. 

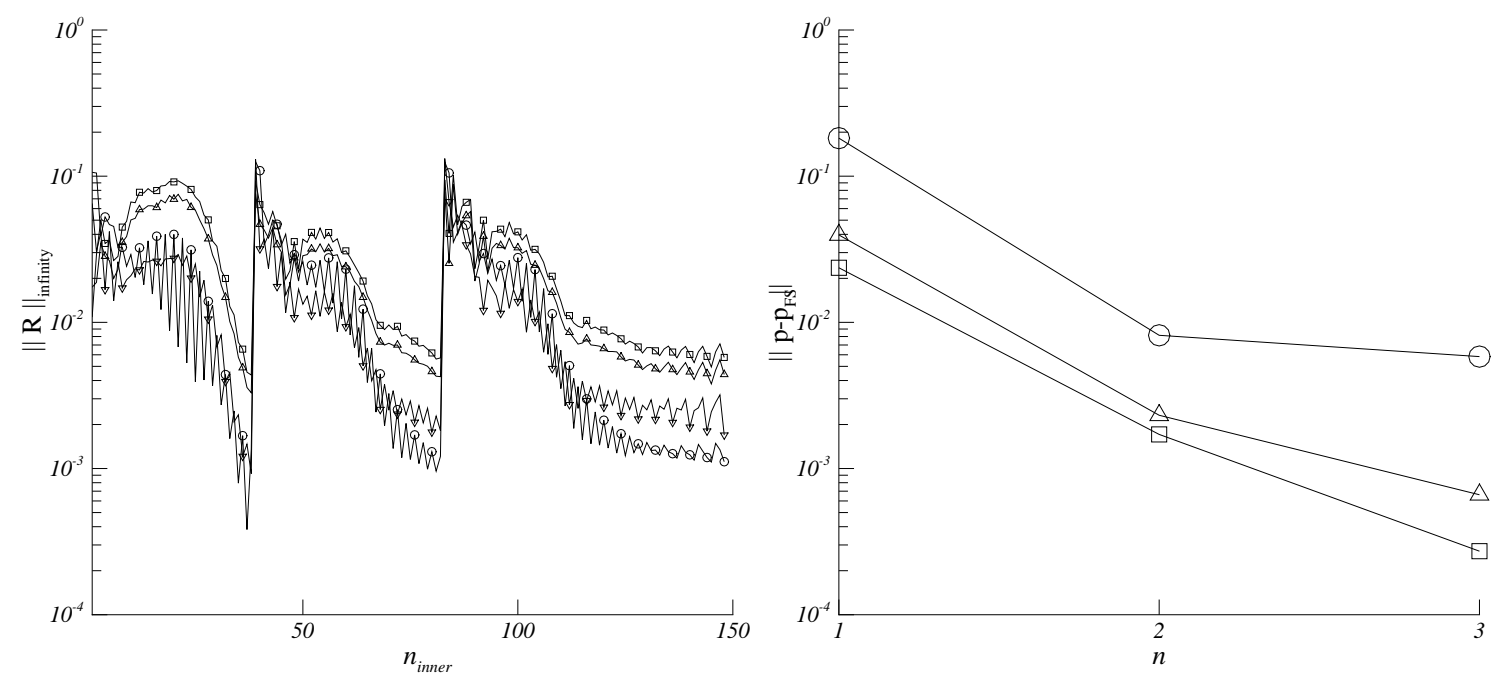

Figure 1: Convergence histories for the Gaussian pressure perturbation with $P=0.05$, on $\Omega_{h}$. Left: of the inner-iteration process, $\mathbf{R}$ is the residual of the continuity equation $(\Delta)$, the $x$-momentum equation $(\square)$, the $y$-momentum equation $(\nabla)$, and the $z$-momentum equation $(\bigcirc)$, only every fourth marker is shown. Right: of the free-surface pressure defect; measured in $L_{1}$-norm $(\square), L_{2}$-norm $(\Delta)$, and $L_{\infty}$-norm $(\bigcirc)$.
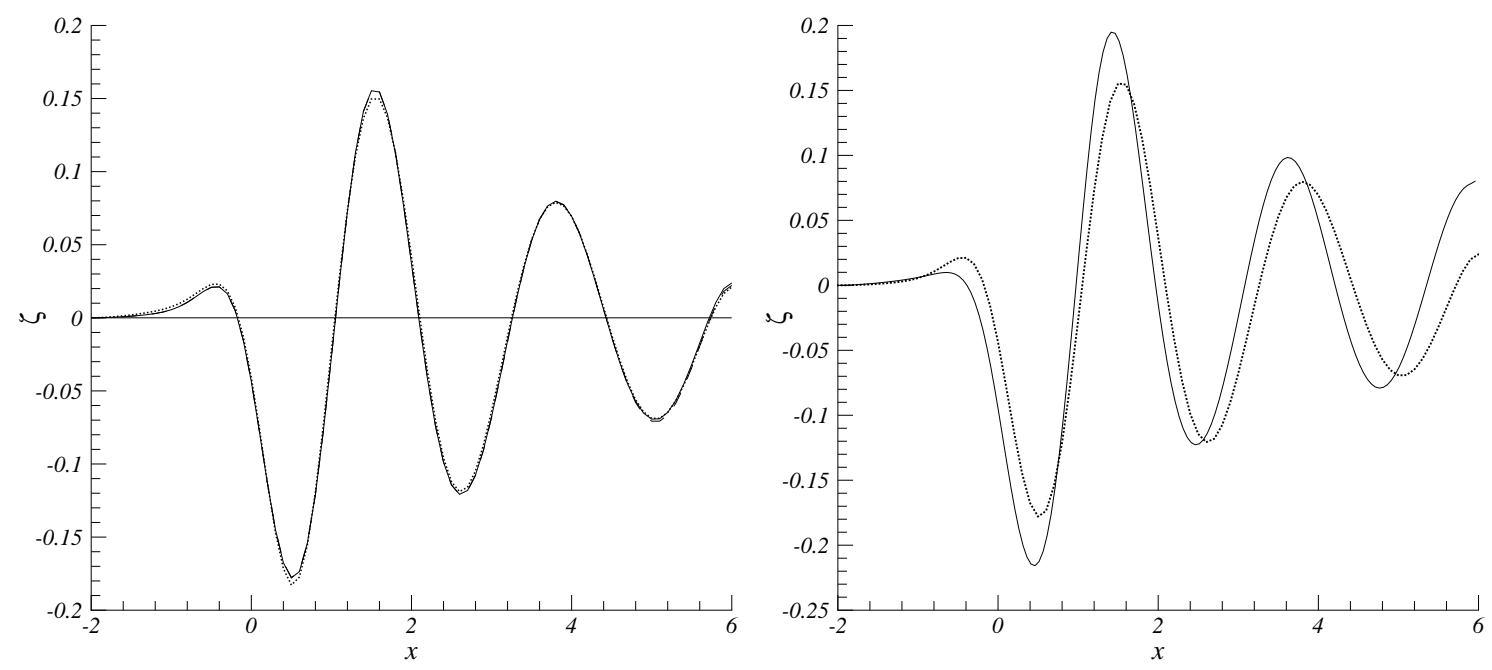

Figure 2: Wave elevations in the plane $y=0$ for the Gaussian pressure perturbation with $P=0.05$. Left: of the present free-surface Navier-Stokes method on $\Omega_{h}$; initial $(\zeta=0), n=1$ (dotted), $n=2$ (dashed), and $n=3$ (solid). Right: of the potential-flow method from ${ }^{9}$ (solid) and the present Navier-Stokes method (dotted). 


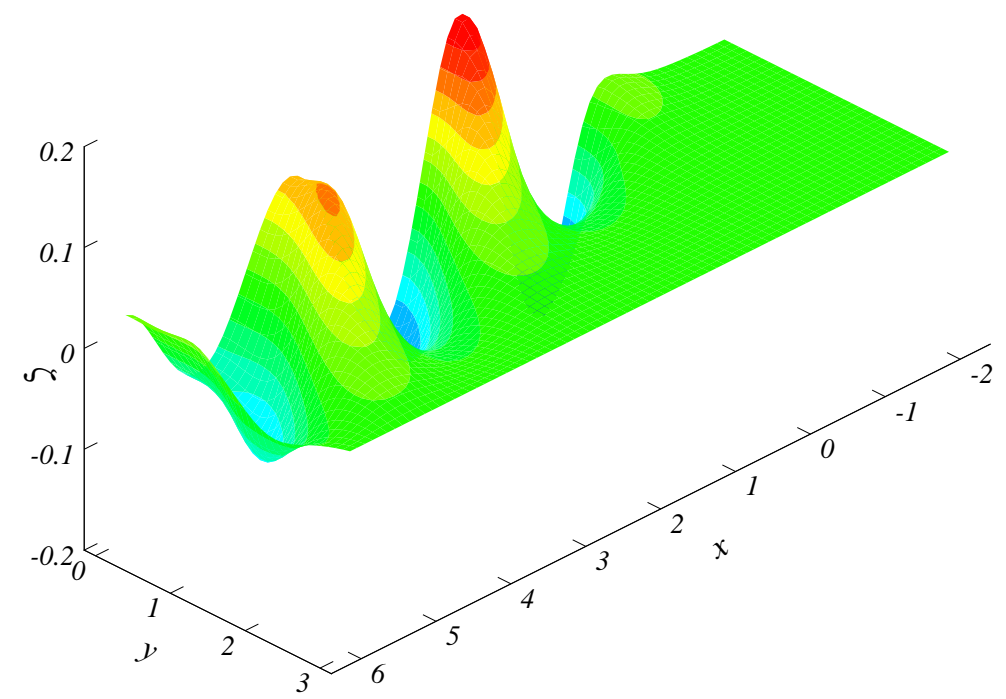

Figure 3: Wave elevation for the Gaussian pressure perturbation with $P=0.05$, on $\Omega_{h}$, after three free-surface updates.
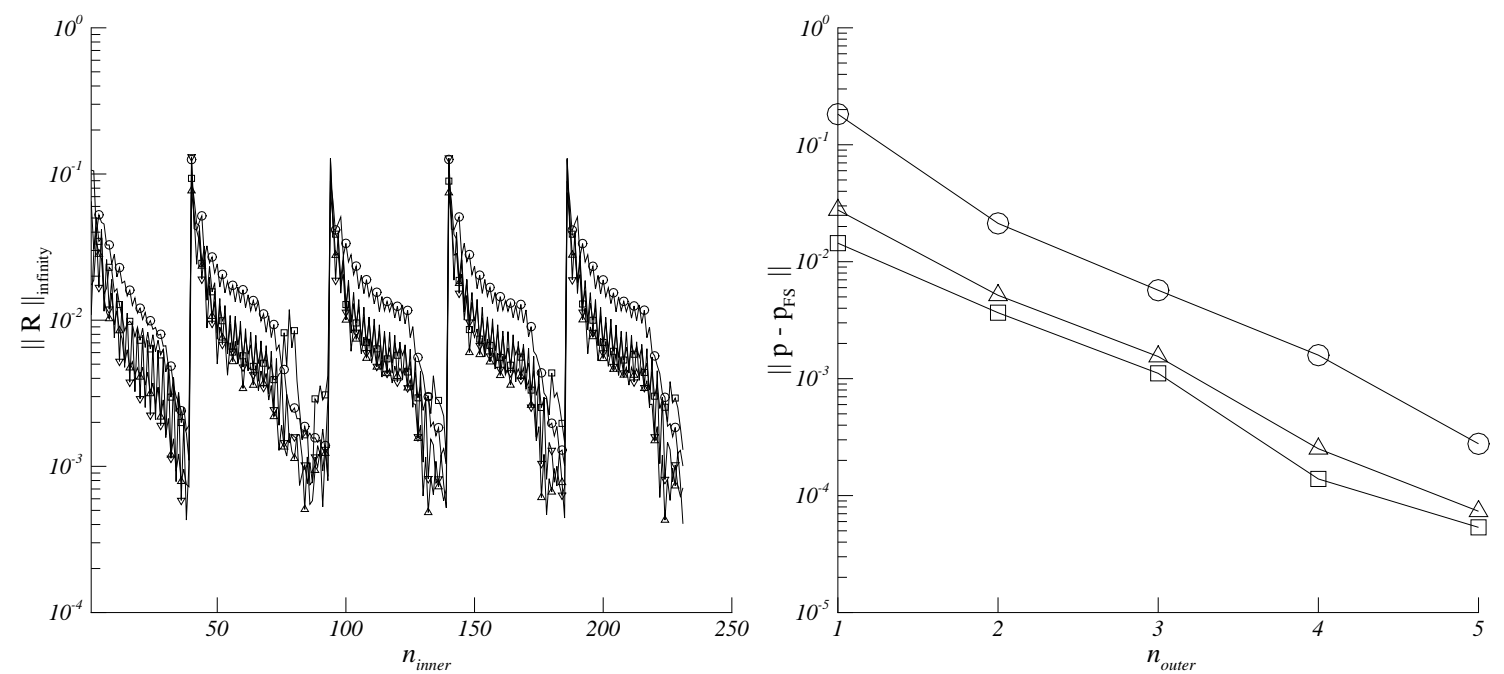

Figure 4: Convergence histories for the Gaussian pressure perturbation with $P=0.05$, on $\Omega_{h}$ with wave-dissipation zone. Left: of the inner-iteration process, $\mathbf{R}$ is the residual of the continuity equation $(\Delta)$, the $x$-momentum equation $(\square)$, the $y$-momentum equation $(\nabla)$, and the $z$-momentum equation $(\bigcirc)$, only every fourth marker is shown. Right: of the free-surface pressure defect; measured in $L_{1}$-norm ( $\left.\square\right), L_{2}$-norm $(\Delta)$, and $L_{\infty}$-norm $(\bigcirc)$.

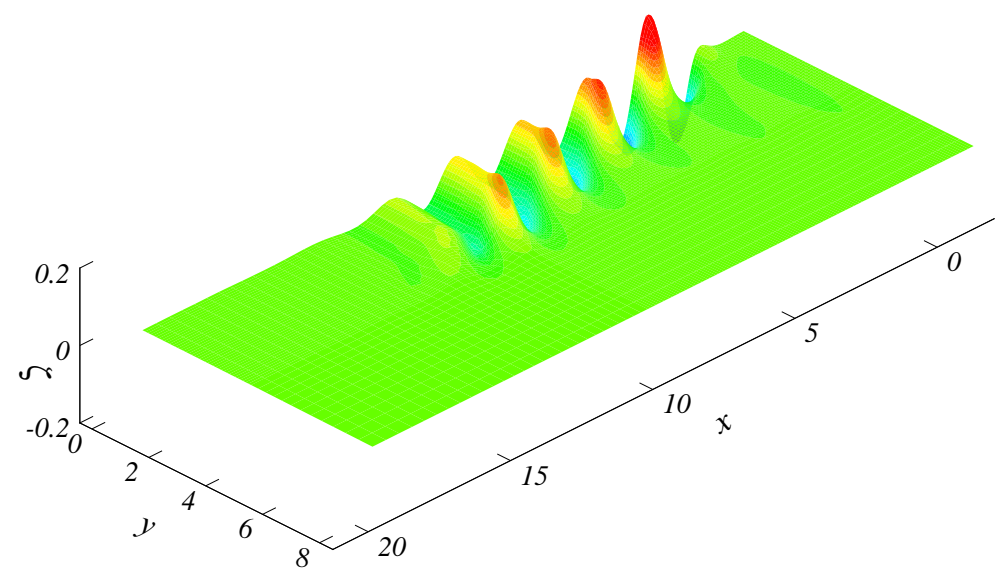

Figure 5: Wave elevation for the Gaussian pressure perturbation with $P=0.05$, on $\Omega_{h}$ with dissipation zone, after five free-surface updates. 

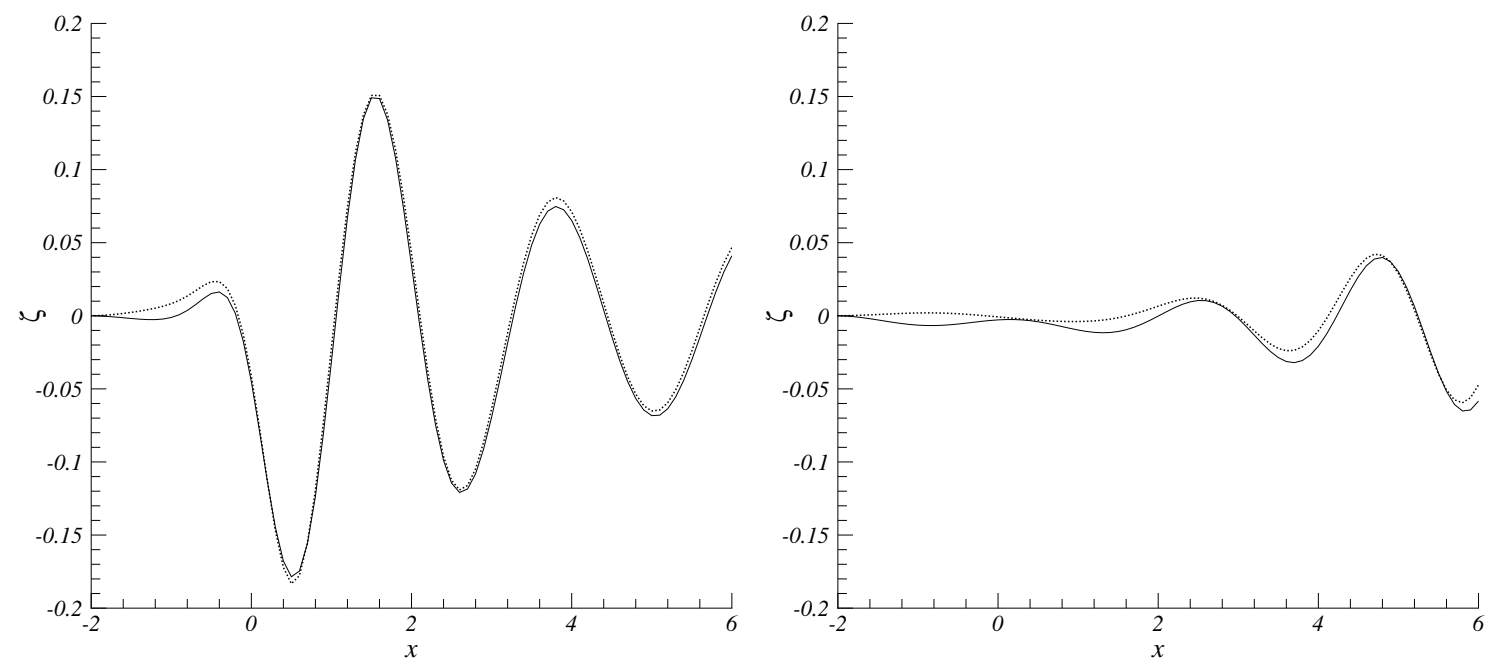

Figure 6: Wave elevation for the Gaussian pressure perturbation with $P=0.05$, on $\Omega_{h}$ with wave-dissipation zone; $n=1$ (dotted) and $n=5$ (solid). Left: In the plane $y=0$. Right: In the plane $y=2$.

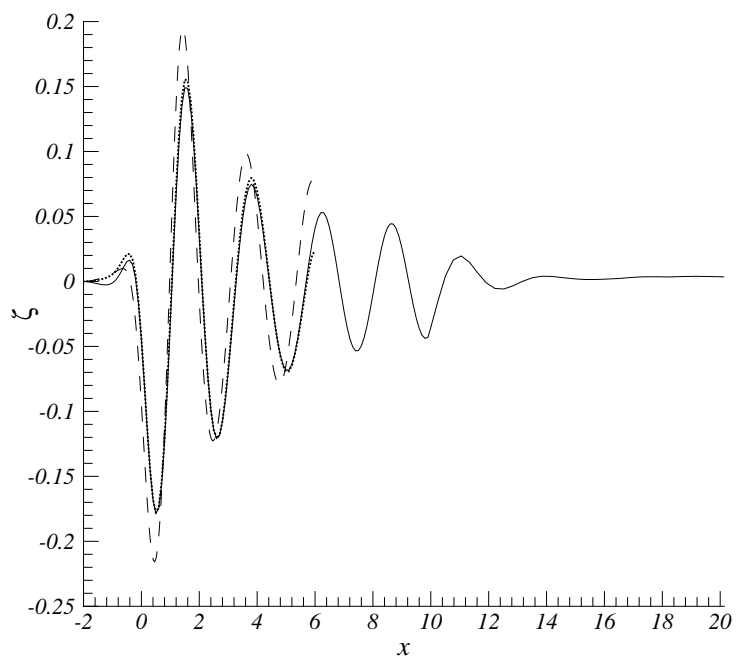

Figure 7: Wave elevation in the plane $y=0$ for the Gaussian pressure perturbation with $P=0.05$; present Navier-Stokes method, on $\Omega_{h}$ with and without wave-dissipation zone (solid and dotted, respectively), and potential-flow method from ${ }^{9}$ (dashed).
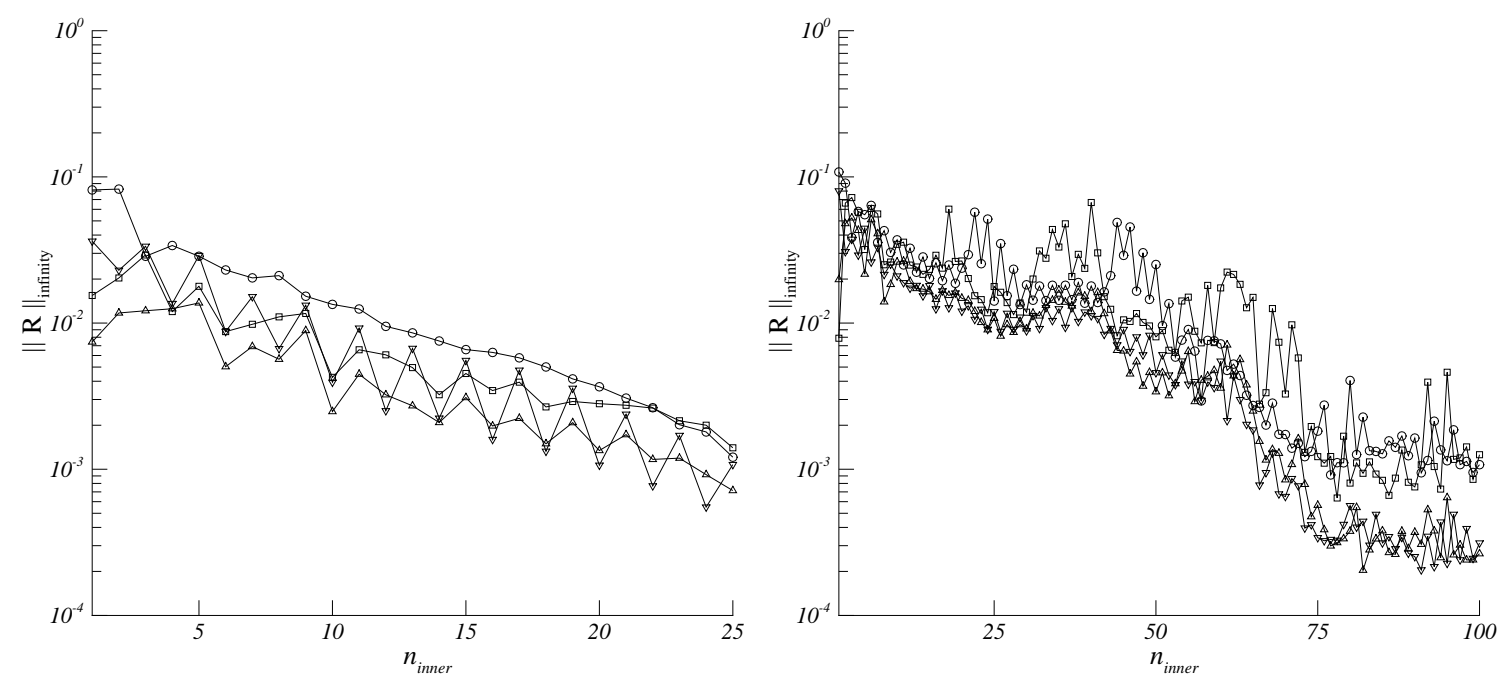

Figure 8: Convergence histories of the inner-iteration process for the Gaussian pressure perturbation with $P=0.05$, with wave-dissipation zone, $\mathbf{R}$ is the residual of the continuity equation $(\Delta)$, the $x$-momentum equation $(\square)$, the $y$-momentum equation $(\nabla)$, and the $z$-momentum equation $(\bigcirc)$, only every fourth marker is shown. Left: on $\Omega_{2 h}$. Right: on $\Omega_{\frac{h}{2}}$. 


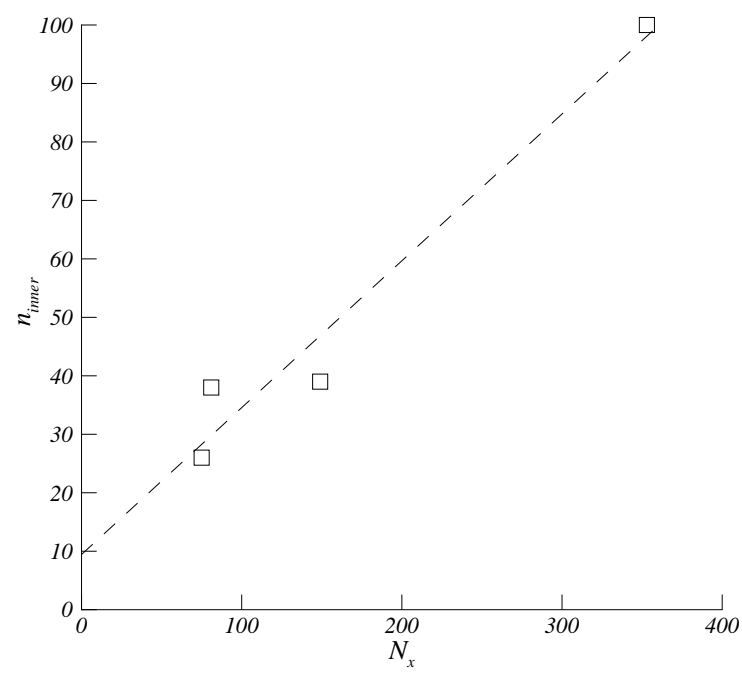

Figure 9: Required number of inner iterations in first Navier-Stokes subproblem versus the number of grid points in $x$-direction.

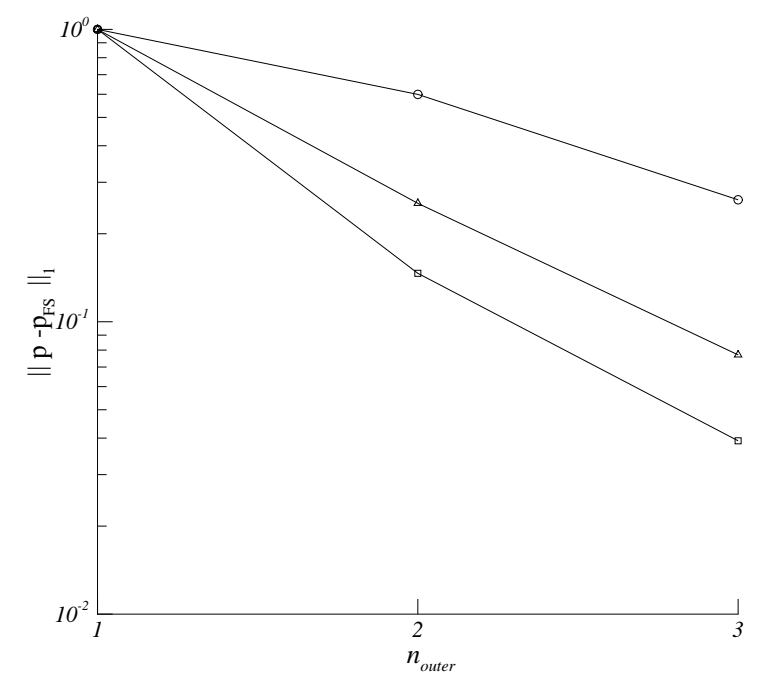

Figure 10: Convergence histories of the outer-iteration process for the Gaussian pressure perturbation with $P=0.05$, with wave-dissipation zone, measured in $L_{1}$-norm; on $\Omega_{2 h}(\square), \Omega_{h}(\Delta)$, and $\Omega_{\frac{h}{2}}(\bigcirc)$.

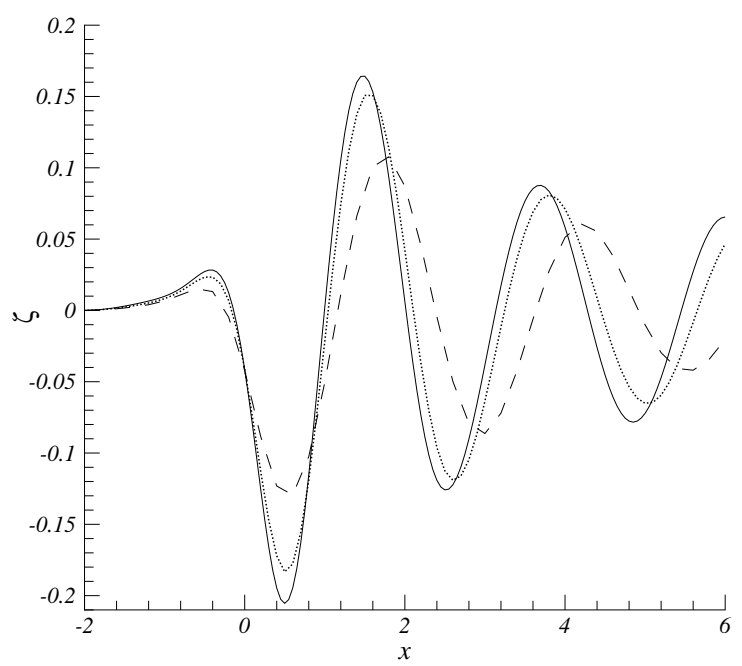

Figure 11: Wave elevation in the plane $y=0$ for the Gaussian pressure perturbation with $P=0.05$; on $\Omega_{2 h}$ (dashed), $\Omega_{h}$ (dotted), and $\Omega_{\frac{h}{2}}$ (solid), all with wave-dissipation zone. 


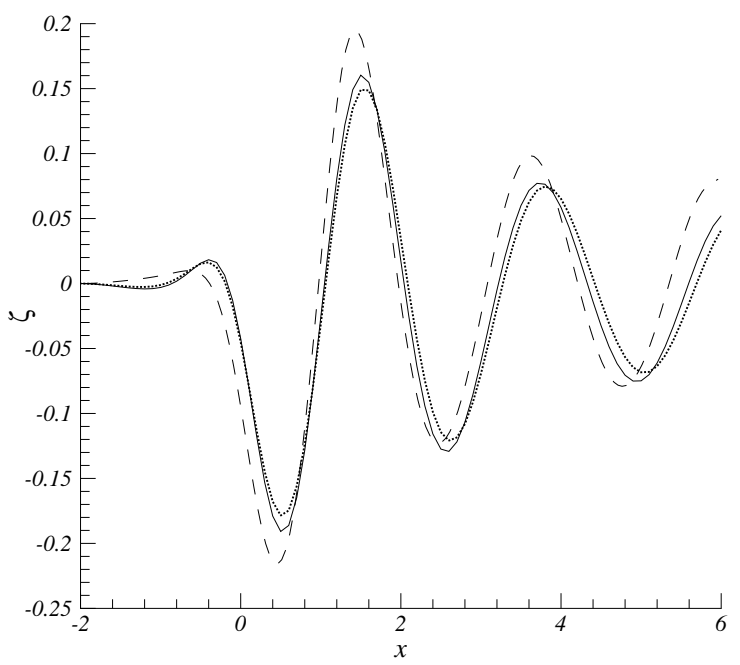

Figure 12: Wave elevation in the plane $y=0$ for the Gaussian pressure perturbation with $P=0.05$; for the Navier-Stokes method on $\Omega_{h}$ with wave-dissipation zone, $\mathcal{O}\left(h^{3}\right)$ scheme (solid) and $\mathcal{O}\left(h^{2}\right)$ scheme (dotted); and for the potential-flow method from ${ }^{9}$ (dashed).
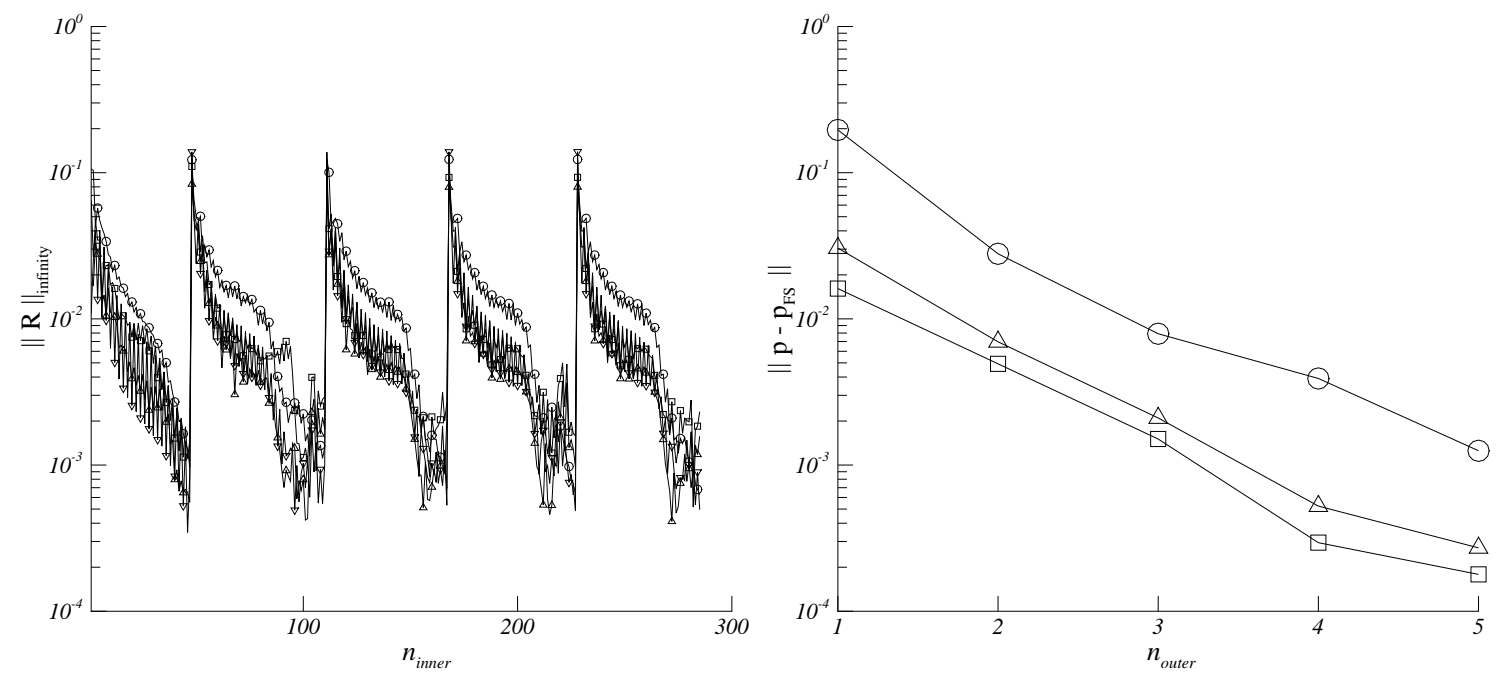

Figure 13: Convergence histories for the Gaussian pressure perturbation with $P=0.05$, on $\Omega_{h}$ with wave-dissipation zone, with $\mathcal{O}\left(h^{3}\right)$ discretization of the quasi free-surface boundary condition. Left: of the inner-iteration process, $\mathbf{R}$ is the residual of the continuity equation $(\Delta)$, the $x$-momentum equation $(\square)$, the $y$-momentum equation $(\nabla)$, and the $z$-momentum equation $(\bigcirc)$, only every fourth marker is shown. Right: of the free-surface pressure defect; measured in $L_{1}$-norm $(\square), L_{2}$-norm $(\Delta)$, and $L_{\infty}$-norm $(\bigcirc)$. 

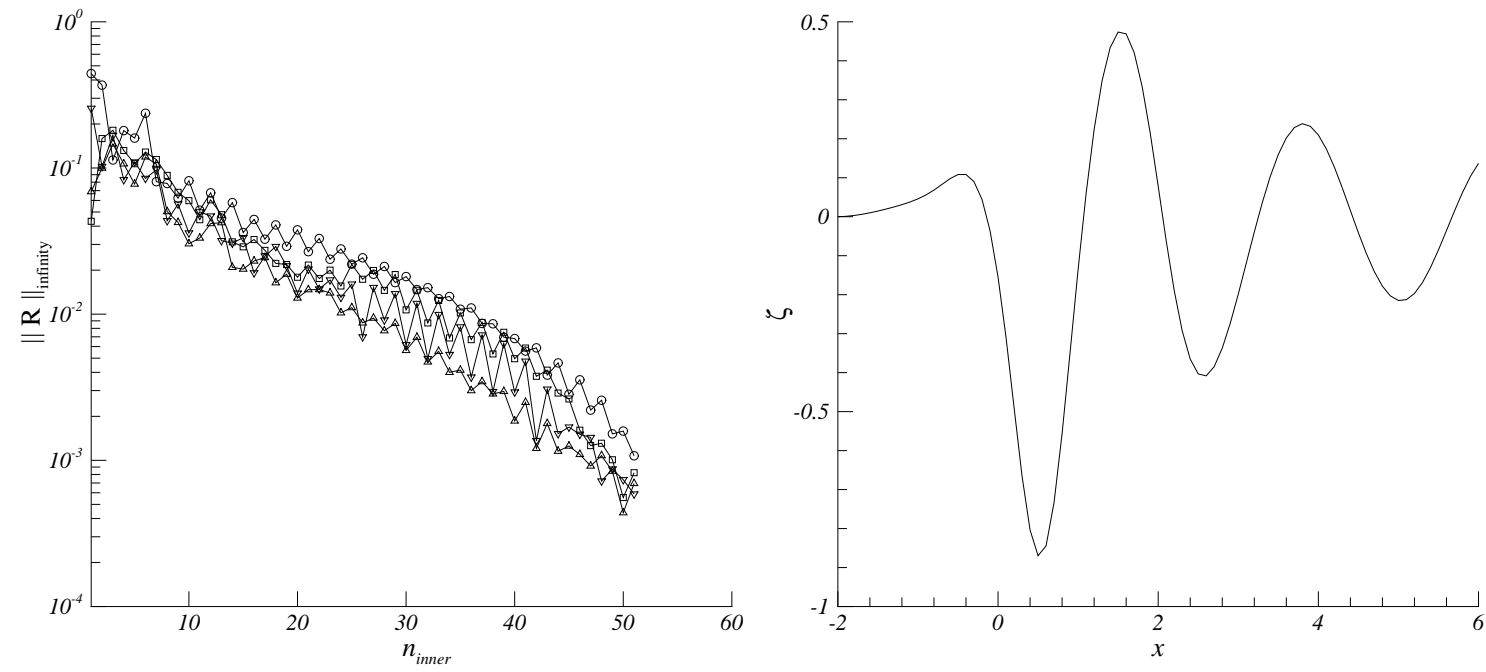

Figure 14: Computational results for the Gaussian pressure perturbation with $P=0.2$, on $\Omega_{h}$ with wave-dissipation zone. Left: convergence history of the inner-iteration process, $\mathbf{R}$ is the residual of the continuity equation $(\Delta)$, the $x$-momentum equation $(\square)$, the $y$-momentum equation $(\nabla)$, and the $z$-momentum equation $(\bigcirc)$, only every fourth marker is shown. Right: wave elevation in the plane $y=0$. 